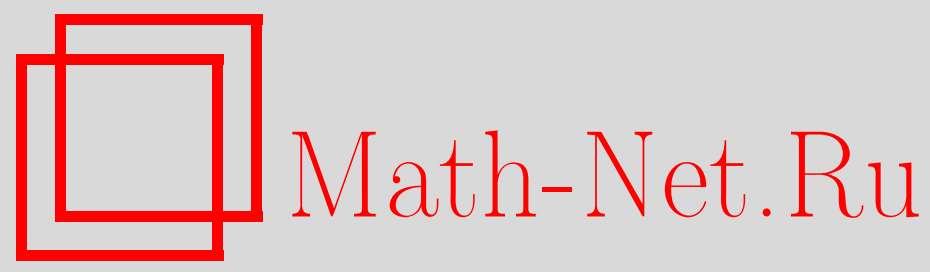

П. М. Ахметьев, Д. Реповш, Й. Малешич, Об инвариантах Милнора четырехкомпонентных зацеплений, Матем. заметки, 2002, том 71, выпуск 4, 496-507

DOI: https://doi.org/10.4213/mzm361

Использование Общероссийского математического портала Math-Net.Ru подразумевает, что вы прочитали и согласны с пользовательским соглашением http://www.mathnet.ru/rus/agreement

Параметры загрузки:

IP: 54.80 .73 .141

26 апреля 2023 г., 15:45:00

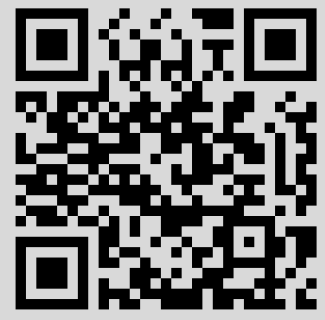




\title{
ОБ ИНВАРИАНТАХ МИЛНОРА ЧЕТЫРЕХКОМПОНЕНТНЫХ ЗАЦЕПЛЕНИЙ
}

\author{
П. М. Ахметьев, Й. Малешич, Д. Реповш
}

\begin{abstract}
В работе исследуется поведение $\mu$-инвариантов Милнора трехкомпонентных и четырехкомпонентных зацеплений относительно дискриминанта, определенного $\Delta$-движениями зацеплений. Определяется новый тип $\Delta$-движений, так называемые сбалансированные $\Delta$-движения, или, коротко, $B \Delta$-движения. Пользуясь тем, что любое четырехкомпонентное зацепление может быть приведено к зацеплению стандартного вида последовательностью сбалансированных $\Delta$-движений, $\Delta$-движений, в которых участвуют не более двух компонент зацепления, и движений Рейдемейстера, $\mu$-инварианты длины 3 для произвольного полуограничивающего четырехкомпонентного зацепления удалось определить аксиоматически.

Библиографоия: 15 названий.
\end{abstract}

1. Введение. В фундаментальной работе Милнора [1] введено понятие целочисленных $\mu$-инвариантов ориентированных многокомпонентных зацеплений с упорядоченными компонентами и, вдобавок, удовлетворяющих дополнительному предположению о полуограничиваемости зацепления. Частичная определенность инвариантов означает, что для определения $\mu$-инварианта порядка $k$ необходимо потребовать равенство нулю всех $\mu$-инвариантов порядков, не превьшающих $k-1$, у произвольного подзацепления, полученного из исходного удалением нескольких компонент. Милноровские $\mu$-инварианты не меняются при изотопиях зацепления. Более того, они не меняются при гомотопиях, при которых компоненты зацепления не пересекаются между собой, но, вообще говоря, самопересекаются.

Инварианты длины 3 оказываются инвариантами конечного порядка (порядка 3 ) в рамках теории Васильева. Существует ровно один $\mu$-инвариант длины 2 в смысле Васильева, которьй определен для произвольного трехкомпонентного зацепления, компоненты которого попарно не зацеплены. Различных инвариантов длины 3 имеется ровно 2 (см. [1]), каж дьй из этих инвариантов определен для четырехкомпонентного полуограничивающего зацепления (см. определение 2.1).

Эквивалентное элементарное определение $\mu$-инвариантов длин 2 и 3 удобно сформулировать на геометрическом языке при помоши понятия поверхности Зейферта. Здесь

Работа выполнена при финансовой поддержке INTAS-CNES, грант № 1048, частичной поддержке Российского фонда фундаментальных исследований, гранты № 99-01-00009, № 96-01-00211 и № 00-05-64015, и Министерства науки и технологии Республики Словения, грант J1-0885-0101-98.

(C) П. М. Ахметьев, Й. МАлешич, Д. Реповш 2002 
используется теорема Портера-Тураева [2], [3], согласно которой милноровские $\mu$-инварианты совпадают с итерированными инвариантами Масси. Инварианты Масси определяются на языке произведений коциклов на дополнении к зацеплению в сфере $S^{3}$ (или даже в произвольной гомологической сфере, как в [3]). Двойственная конструкция использует лишь геометрические свойства поверхностей Зейферта, она была открыта Кохраном в [4].

Никакой милноровский инвариант длины 2 или 3 не удается доопределить до целочисленного инварианта гомотопии произвольных зацеплений, как инвариант конечного порядка в смысле Васильева (см. [5]). Если отказаться от первого условия, т.е. не требовать инвариантности при гомотопиях, а требовать лишш конечности порядков инвариантов, то подобное обобщение, вообще говоря, возможно. Простейший пример милноровского инварианта, которьй является инвариантом порядка 3 и определен на классах изотопии произвольного ориентированного (неупорядоченного) двукомпонентного зацепления, доставляет обобщенный инвариант Сато-Левина (см. [6], [7]). Изучение инварианта Сато-Левина для двукомпонентного зацепления с нулевьм коэффициентом зацепления, как милноровского $\bar{\mu}$-инварианта, происходит в [8].

В общем случае проблема доопределения целочисленного милноровского $\mu$-инварианта до инварианта изотопического класса зацепления общего вида с сохранением порядка инварианта в смысле Васильева очень важна для решения прикладных задач из физики; в простейшей постановке (без использования конструкции Васильева) она рассматривалась в [9]. Для инварианта порядка 2 такого обобщения построить не удается, хотя соответствующая теорема о запрете, по-видимому, не доказана. Напротив, оба инварианта порядка 3 допускают подобное обобщение, эта теорема будет доказана во второй части работы, рукопись которой готовится к публикации.

Для решения этой задачи мы определяем инварианты порядка 3 аксиоматически, используя новое понятие $\Delta$-движения проекции зацепления, так называемое сбалансированное $\Delta$-движение. Понятие $\Delta$-движения, как преобразование одного зацепления в другое, впервые введено в работе [10]. Оно активно изучалось в последние годы с различных точек зрения (см. [11]-[13]). При изучении $\mu$-инвариантов старших порядков возникают естественные обобщения этого понятия. Например, в [13, с. 58] инварианты порядка 3 изучаются при помоши обобщенного $\Delta$-движения, в окрестности особой точки которого располагаются все четыре компоненты зацепления, при этом движении инвариант порядка 3 меняется на \pm 1 .

В основе нашего подхода лежит другое обобщение. С одной стороны, сбалансированное $\Delta$-движение (далее для краткости мы иногда будем употреблять термин $B \Delta$-движение) сохраняет класс полуограничивыемых зацеплений. С другой стороны, пространство полуограничиваемых зацеплений становится связным, если разрешено переходить от одного зацепления к другому при помощи $B \Delta$-движения. Теперь, если известно, как меняются инварианты порядка 3 при сбалансированных $\Delta$-движениях, удается определить их значение для любого полуограничиваемого зацепления. При этом полагают, что значения инвариантов на зацеплении с компонентами, заключенными в непересекающиеся шары, равны 0. В части II работы, готовящейся к печати, при помощи этого подхода мы обобщим определение $\mu$-инвариантов порядка 3 и определим их для произвольного четырехкомпонентного зацепления без предположения о его полуограничиваемости. 
Остановимся подробнее на содержании настоящей работы. В п. 2 мы даем новое определение полуограничивающего трех- и четырехкомпонентного зацепления, которое отличается от данного выше тем, что каждая компонента зацепления не предполагается связной. Мы формулируем понятие бордизма многокомпонентного зацепления, (которое, как подмечено в [9], моделирует процесс перезамыкания силовых линий магнитного поля для среды с высокой магнитной проводимостью). Это новое понятие является аналогом общепринятого понятия гомотопии зацеплений, последнее изучено значительно лучше.

Далее в п. 3 мы переходим к изучению трехкомпонентных зацеплений (со связными компонентами), напоминаем определение $\mu$-инварианта порядка 2 и доказьваем его основные свойства. В заключение этого пункта мы иллюстрируем наш подход к изучению инвариантов порядка 3 на более простом примере, вводя понятие сбалансированного $\times$-движения, которое сохраняет класс полуограничивающих трехкомпонентных зацеплений. При этом пространство трехкомпонентных полуограничивающих зацеплений становится связным. Заметим, что при помоши сбалансированных $\times$-движений доопределить инварианты порядка 2 до полных не удается.

В п. 4 мы формулируем теорему, согласно которой пространство четырехкомпонентных полуограничивающих зацеплений (со связными компонентами) состоит из единственного класса эквивалентности зацеплений относительно $B \Delta$-преобразований. Напоминается определение инвариантов порядка 3 , доказываются их основные свойства. Формулируется правило подскока $\mu$-инварианта при $B \Delta$ - движении. Последний п. 5 посвящен доказательствам.

Большую помощь при обсуждениях нам оказали С. А. Мелихов, В. П. Лексин и А. И. Рез, которым мы искренне благодарны.

2. Полуограничивающие (в обобщенном смысле) многокомпонентные зацепления и проблема их классификации. Пусть $I=\{1, \ldots, s\}$ - отрезок натурального ряда; в основном рассматриваются два случая, $s=2,3$. Пусть $L \subset \mathbb{R}^{3}-$ замкнутое гладкое одномерное ориентированное подмногообразие, каждой компоненте которого (возможно, несвязной или даже пустой) поставлено в соответствие натуральное число из $I$. Подмногообразие $L$ назьвается многокомпонентным (в обобщенном смысле) зачеплением. Компоненты (вообще говоря, несвязные) обобщенного многокомпонентного зацепления будем обозначать через $L_{i}, i \in I$. Для краткости в этом пункте мы будем опускать слово обобщенное. Воспользуемся стандартным понятием кобордизма вложенных подмногообразий. В нашем случае кобордизм можно представлять себе как последовательность регулярных гомотопий вложенных подмногообразий и морсовских перестроек или пересоединений компонент (см. [9]). При перестройках сохраняется как ориентация на компонентах, так и значение из $I$.

Пусть $L_{i}$ - подмногообразие, соответствующее выделенному значению $i \in I$. Компоненты связности этого подмногообразия будем в этом пункте обозначать вторым индексом $j, j \in\left\{1, \ldots, s_{i}\right\}$, например: $L_{2,4} \subset L_{2} \subset L$.

ОПРЕДЕЛЕниЕ 2.1. Произвольное двух- или трехкомпонентное зацепление называется полуограничивающим, если для любых двух пар индексов $i, j, i \in\{1,2\}$ или $i \in$ $\{1,2,3\}, j \in\left\{1, \ldots, s_{i}\right\}$, справедливо равенство

$$
\operatorname{lk}\left(L_{i_{1}, j_{1}} ; L_{i_{2}, j_{2}}\right)=0 .
$$


Произвольное четырехкомпонентное зацепление называется полуограничивающим, если вьполнены два условия. Во-первых, для любых двух пар индексов $i, j, i \in\{1,2$, $3,4\}, j \in\left\{1, \ldots, s_{i}\right\}$, справедливо равенство

$$
\operatorname{lk}\left(L_{i_{1}, j_{1}} ; L_{i_{2}, j_{2}}\right)=0 .
$$

Во-вторых, для любых трех пар индексов $i, j$ справедливо равенство

$$
\mu\left(L_{i_{1}, j_{1}} ; L_{i_{2}, j_{2}} ; L_{i_{3}, j_{3}}\right)=0
$$

где через $\mu$ обозначен милноровский целочисленный инвариант длины 2 , определение которого напоминается в п. 3 .

ОПРедЕлЕниЕ 2.2. Два $s$-компонентных полуограничивающих зацепления $L$ и $L^{\prime}$, $s=2,3,4$, называются бордантными, если они бордантны в классе полуограничивающих зацеплений. Другими словами, если для каждого пересоединения компонент при бордизме свойство полуограничиваемости сохраняется.

Используя элементарные соображения, изложенные в [9], легко доказать, что два многокомпонентных зацепления общего вида бордантны (без предположения о полуограничиваемости), если симметрические матрицы размера $s \times s$, составленные из сумм (по паре вторых индексов) коэффициентов зацепления каждой пары компонент с данной парой первых индексов, совпадают. Свойства бордизма при условии полуограничиваемости сложнее. Те $s$-компонентные зацепления, матрица попарных коэффициентов зацепления компонент которых нулевая, образуют подпространство, особыми элементами которого (т.е. дискриминантом) служат компоненты, образующиеся в процессе одного или нескольких пересоединений. Под пересоединением здесь понимается морсовская критическая точка проекции многообразия, осуществляющего кобордизм, на ось времени (подробнее см. [9]). Вопрос о классификации инвариантов Васильева для рассматриваемой теории, по-видимому, не изучался. С другой стороны, нетривиальные в этой теории инварианты заведомо существуют. Простейший пример доставляется обобщенным инвариантом Сато-Левина.

Вопрос об изучении подобных инвариантов продиктован проблемой изучения и классификации бездивергентных векторных полей. Предположим, что поле представлено как объединение $s$ подполей с непересекаюшимися носителями (каждое слагаемое в этой сумме моделирует объединение некоторого числа магнитных трубок). Требуется изучить поведение того или иного инварианта при эволюции системы магнитных трубок при наличии слабого пересоединения траекторий поля. Например, важно предсказывать статистические свойства пространственного спектра спиральности поля (т.е. интенсивность поля в областях пространства, где преобладает тот или иной знак коэффициентов зацепления магнитных трубок), если известна информация о поведении высших инвариантов зацепленности силовых линий.

В рамках нашей работы мы ограничимся далее стандартным понятием многокомпонентного зацепления, подразумевая, что каждая компонента зацепления связна и не пуста. Предположительно, наши построения переносятся на случай полуограничивающих многокомпонентных (в обобщенном смысле) зацеплений, что доставляет информацию об инвариантах конечного порядка в вьшеизложенной теории. Например, значения соответствующих $\mu$-инвариантов для этих зацеплений можно определить по аддитивности. Для изучения свойств определенньх таким образом инвариантов при бордизмах требуются дополнительные исследования. 
3. Трехкомпонентные полуограничивающие зацепления. Пусть $L=L_{1} \cup$ $L_{2} \cup L_{3}$ - трехкомпонентное полуограничивающее зацепление со связными и непустыми компонентами. Пусть $S_{1}, S_{2}$ и $S_{3}$ - поверхности Зейферта, принадлежащие компонентам $L_{1}, L_{2}$ и $L_{3}$ зацепления, такие, что $L_{i} \cap S_{j}=\varnothing$ для $i \neq j$. Пересечение $S_{1} \cap S_{2} \cap S_{3}$ состоит из конечного числа точек. Фиксируем порядок компонент (т.е. перестановку $i j k$ индексов 1,2 и 3 ). Напомним, что компоненты зацепления снабжены ориентацией, следовательно, поверхности Зейферта также ориентированы в соответствии со стандартным правилом согласования ориентации многообразия и его края. Это позволяет однозначно определить знак в каждой точке пересечения поверхностей в соответствии со стандартным правилом выбора ориентации на подмногообразии трансверсального пересечения нескольких упорядоченных экземпляров подмногообразий в ориентированном объемлющем многообразии. Определим значение $\mu_{i j k}(L)$ как сумму знаков по всем точкам пересечения.

ПРЕДЛОЖЕНИЕ 3.1. Значение $\mu_{i j k}(L)$ корректно определено, т.е. не зависит от выбора поверхностей Зейферта. Оно меняет знак при каждой перемене ориентации и при перемене четности перестановки $i j k$.

ЗАмЕчАнИЕ. Предложение 3.1 , а также соответствие так определенного $\mu_{i j k}$ с его алгебраическими определениями [14] было установлено в [8] (см. также [4]).

В качестве примера рассмотрим два борромеевских зацепления, которые изображены на рис. 1.
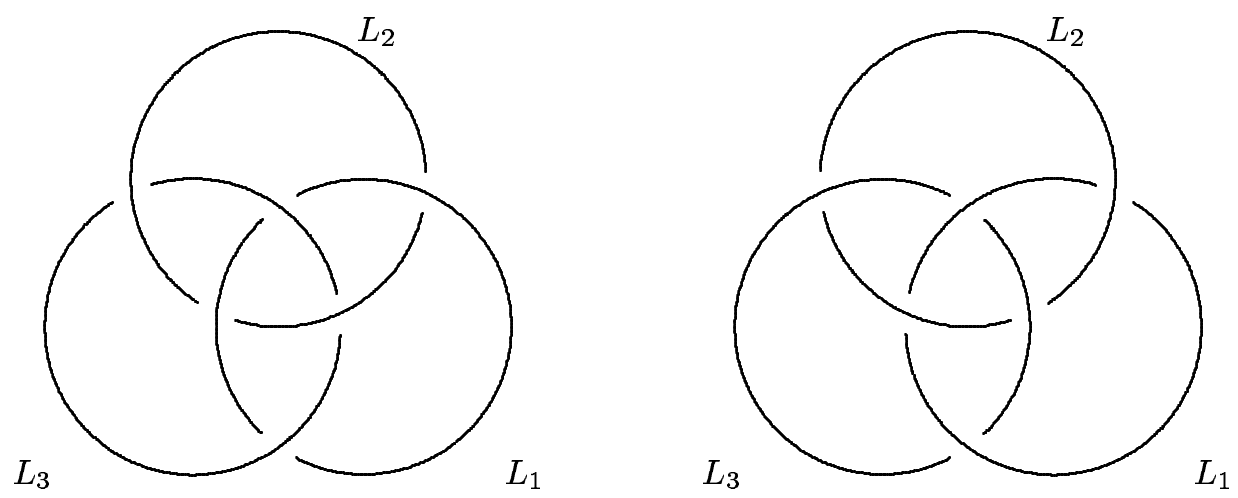

Рис. 1. Борромеевские зацепления

Вычислим инвариант $\mu_{123}(L)$ для зацепления слева, выбрав положительную ориентацию на проекции каждой компоненты и указанный порядок $L_{1}, L_{2}, L_{3}$. Следуя Гауссу, принято изучать расположение прообразов точек самопересечения прекции зацепления, учитывая при этом их порядок вдоль оси проекции (левая часть рис. 2).

Каждая стрелка связывает точки, отображенные при проекции в двойную точку, направление стрелки указьвает на точку, лежащую ниже. Аналогично рассмотрим диски $\widehat{S}_{1}, \widehat{S}_{2}$ и $\widehat{S}_{3}$, границами которых являются $L_{1}, L_{2}$ и $L_{3}$. Диски попарно пересекаются в отрезках $\left[a_{i} b_{i}\right], i=1,2,3$. Стрелки связывают пары отрезков, отображающихся на один отрезок. 

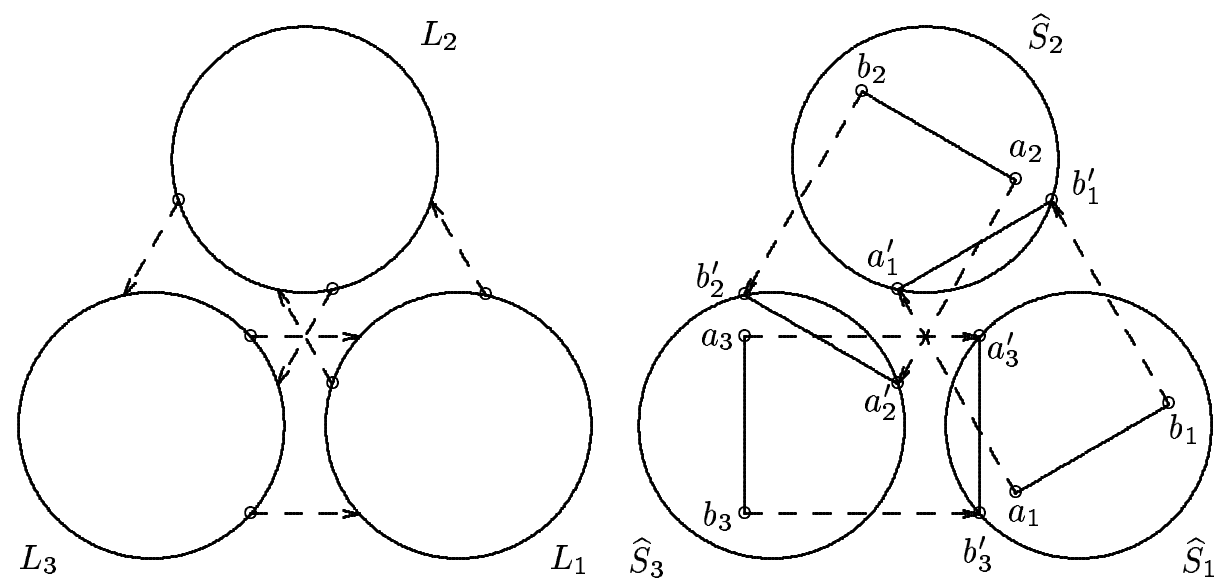

Рис. 2. Диаграммы Гаусса

Чтобы получить поверхности Зейферта $S_{1}, S_{2}$ и $S_{3}$, удовлетворяющие условию $S_{i} \cap L_{j}$ $=\varnothing$ для $i \neq j$, перестроим диски $\widehat{S}_{1}, \widehat{S}_{2}$ и $\widehat{S}_{3}$. Из $\widehat{S}_{1}$ вырежем два маленьких диска с центрами $a_{1}$ и $b_{1}$ и приклеим трубочку, концентрическую дуге $a_{1}^{\prime} b_{1}^{\prime}$, лежащей на окружности $L_{2}$. Аналогичными приклейками трубочек перестроим $\widehat{S}_{2}$ и $\widehat{S}_{3}$. (В общем случае поверхность Зейферта строится методами, описанными в [4].)

Алгебраическую сумму точек пересечения $S_{1} \cap S_{2} \cap S_{3}$ вычислим как алгебраическую сумму точек пересечения поверхности $S_{3}$ и кривой $S_{1} \cap S_{2}$ или, что то же самое, как коэффициент зацепления ориентированных кривых $L_{3}$ и $S_{1} \cap S_{2}$. Замкнутую кривую $S_{1} \cap S_{2}$ составляют отрезок $\left[b_{1} a_{1}\right]$, лежащий на диске, и дуга $\left[a_{1} b_{1}\right]$, лежащая на трубочке. Коэффициент зацепления равен 1 , следовательно, $\mu_{123}(L)=1$. Таким же образом для борромеевского зацепления, которое показано на рис. 1 справа, $\mu_{123}(L)=-1$.

Мураками и Наканиши был определен специальньй тип перестройки зацепления, которое называется $\Delta$ - движсением (см. [10]). На проекции зацепления это преобразование выглядит как фальшивый вариант движения Рейдемейстера, оно не соответствует проекции изотопии зацепления. Это движение изображено на рис. 3 , центральная и левая картинки.

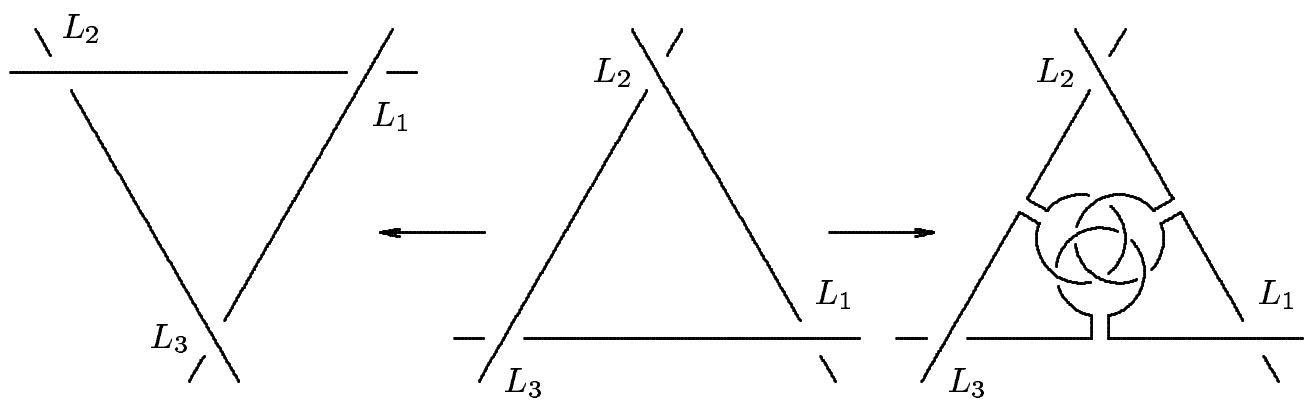

Рис. 3. $\Delta$-движение 
Центральная картинка назьвается исчезающим треугольником. Определим знак исчезаюшего треугольника, стороны которого лежат на разных компонентах зацепления, согласно следующему правилу. Сначала пусть $\rho-$ это число тех сторон, у которых ориентация противоположна положительной ориентации треугольника на плоскости (т.е. против часовой стрелки), определим $\varepsilon_{1}=(-1)^{\rho}$. Пусть $\varepsilon_{2}$ - четность перестановки индексов 1,2 и 3 , определенной положительной ориентацией треугольника (т.е. +1 в случае четной перестановки и -1 в случае нечетной). Пусть $x-$ двойная точка на проекции зацепления, $\xi$ и $\eta$ - касательные векторы в точке $x$, причем $\xi$ касается верхной ветви. Знак $\mathscr{O}(x)$ точки $x$ определим как знак репера $(\xi, \eta)$. Произведение знаков вершин исчезающего треугольника не зависит от выбора ориентаций компонент - оно определяет его mun. Это произведение обозначим через $\varepsilon_{3}$. Наконец, произведение $\varepsilon=\varepsilon_{1} \varepsilon_{2} \varepsilon_{3}$ назовем знаком исчезающего треугольника.

Поскольку при $\Delta$-движении знаки двойных точек не меняются, коэффициенты зацепленности компонент также не меняются. Таким образом, любое полуограничивающее зацепление остается полуограничивающим и после $\Delta$-движения.

Следующее предложение было недавно доказано в работе [11] в рамках алгебраического подхода. Наше доказательство, используюшее геометрию поверхностей Зейферта, существенно короче.

ПРЕДЛОЖЕНИЕ 3.2. При $\Delta$-движении инвариант $\mu_{i j k}$ увеличивается на знак исчезающего треугольника.

ДокАЗАТЕЛЬСтво. Как видно на рис. 3 (правая картина), $\Delta$-движение может быть реализованопрямой суммой борромеевского зацепления внутри исчезающего треугольника и трех последовательных движений Рейдемейстера, при каждом из которых на проекции возникает или изчезает пара близких точек самопересечения, в которых функция $\mathscr{O}$ имеет различные значения. Исчезающий треугольник в центре борромеевского зацепления должен быть таким же, как внешный треугольник. Ввиду того, что новая поверхность Зейферта получается просто приклейкой поверхности Зейферта борромеевского зацепления, инвариант $\mu$ увеличится на знак исчезающего треугольника в центре борромеевского зацепления.

В заключительной части этого пункта рассмотрим построения, не использующиеся непосредственно при доказательстве главного результата о $\mu$-инвариантах порядка 3 , которое изложено в следующем пункте. Тем не менее, эти построения хорошо иллюстрируют основную идею нашего подхода, являясь при этом более простыми.

Введем специальное движение, которое будем назьвать сбалансированным х-движением. В результате сбалансированного $\times$-движения проекция зацепления вообще не меняется, а изменяется значение функции $\mathscr{O}$ в паре точек, лежаших на пересечении пары одинаковых компонент на проекции. При этом значения функции $\mathscr{O}$ в рассматриваемой паре точек должны различаться. Очевидно, произвольное сбалансированное ×-движение не меняет коэффициента зацепления компонент. В частности, оно переводит полуограничивающее зацепление в полуограничивающее.

По определению в $B \times$-движении участвуют только две компоненты, образующие пару двуугольников. Знаки вершин каждого двуугольника всегда различаются.

Пусть $i j k$ - перестановка индексов 1,2 и 3. Предположим, что в данном сбалансированном $\times$-движении участвуют компоненты $L_{i}$ и $L_{j}$. Обозначим через $L_{i}^{+}$и $L_{j}^{+}$те ветви 
кривых $L_{i}$ и $L_{j}$, у которых до момента преобразования начало находится в положительной вершине, а конец - в отрицательной с учетом ориентации компонент. Аналогично, через $L_{i}^{-}$и $L_{j}^{-}$обозначим дополнительные ветви.

ПРЕДЛОЖЕНИЕ 3.3. При сбалансированном $\times$-движсении полуограничивающего трехкомпонентного зачепления $L=L_{1} \cup L_{2} \cup L_{3}$ значение инварианта $\mu_{i j k}(L)$ увеличивается на коэффициент зачепления $\operatorname{lk}\left(L_{i}^{+} \cup L_{j}^{-}, L_{k}\right)$.

ДокАЗАТЕЛЬСтво. Пусть $S_{i}$ и $S_{j}$ - поверхности Зейферта кривых $L_{i}$ и $L_{j}$ соответственно, которые удовлетворяют условию $S_{i} \cap L_{j}=S_{j} \cap L_{i}=\varnothing$ в начальный момент времени сбалансированного $\times$-движения. Чтобы получить поверхности Зейферта $\widehat{S}_{i}$ и $\widehat{S}_{j}$, удовлетворяющие аналогичному условию послепреобразования зацепления, надо перестроить $S_{i}$ и $S_{j}$, вырезав по паре дисков и добавив по трубочке к каждой из поверхностей. Легко проверить, что кривые пересечения $\widehat{S}_{i} \cap \widehat{S}_{j}$ и $S_{i} \cap S_{j}$ различаются только на одну замкнутую компоненту $K$, для которой $\operatorname{lk}\left(K, L_{k}\right)=\operatorname{lk}\left(L_{i}^{-} \cup L_{j}^{+}, L_{k}\right)$. Следовательно, значение инварианта $\mu_{i j k}(L)$ увеличивается на число $-\operatorname{lk}\left(L_{i}^{-} \cup L_{j}^{+}, L_{k}\right)$, которое из-за равенств $\operatorname{lk}\left(L_{i}, L_{k}\right)=\operatorname{lk}\left(L_{j}, L_{k}\right)=0$ равно числу $\operatorname{lk}\left(L_{i}^{+} \cup L_{j}^{-}, L_{k}\right)$.

Пользуясь доказанным предложением, можно предложить следующий способ аксиоматического построения инварианта $\mu_{i j k}$ для произвольного трехкомпонентного полуограничивающего зацепления. Легко видеть, что пространство полуограничивающих трехкомпонентных зацеплений становится связньп после добавления особых элементов, соответствуюших сбалансированным $\times$-движениям. Определим значение $\mu_{i j k}$ равным нулю на произвольном зацеплении, компоненты которого заключены в непересекающиеся шары. Изменение инварианта при сбалансированном $\times$-движении происходит согласно правилу, сформулированному в предложении 3.3. Теперь доказательство предложения превращается в доказательство корректности инварианта с требуемыми свойствами, а единственность такого инварианта вытекает из способа его построения.

4. Четырехкомпонентные полуограничивающие зацепления. Пусть $L=$ $L_{1} \cup L_{2} \cup L_{3} \cup L_{4} \subset \mathbb{R}^{3}$ - ориентированное четырехкомпонентное полуграничивающее зацепление со связными непустыми компонентами. Для каждой перестановки $i j k l$ индексов $\{1,2,3,4\}$ однозначно определен инвариант Милнора $\mu_{i j k l}(L) \in \mathbb{Z}$ (cм. [1]). Среди инвариантов, порожденных различными подстановками, лишь два являются независимыми, например: $\mu_{1234}$ и $\mu_{1342},-$ остальные инварианты через них выражаются.

Сформулируем определение инвариантов $\mu_{i j k l}$ при помощи семейства поверхностей Зейферта. В предложении 4.2 сформулируем и докажем свойства построенных инвариантов и далее перейдем к изучению этих инвариантов при помощи сбалансированных $\Delta$-движений.

Пусть $S_{1}, S_{2}, S_{3}, S_{4}$ - семейство поверхностей Зейферта к каждой из компонент, причем для произвольных $i, j, l, k$ вьполнены следующие геометрические равенства:

$$
S_{i} \cap L_{j}=S_{j} \cap L_{k}=S_{j} \cap S_{l}=\varnothing .
$$

Существование семейства поверхностей с указанньм свойством вытекает из определения полуограничиваемости зацепления. Если поверхности Зейферта находятся в общем положении, то кривые их попарных трансверсальных пересечений $\Gamma_{i, j}=S_{i} \cap S_{j}$, $\Gamma_{k, l}=S_{k} \cap S_{l}$ являются вложенными и не пересекаются. 
ОПРЕДЕЛЕниЕ 4.1. Определим значение инварианта $\mu_{i j k l}$ как разность коэффициентов зацеплений по формуле

$$
\operatorname{lk}\left(\Gamma_{i k} ; \Gamma_{j l}\right)-\operatorname{lk}\left(\Gamma_{i l} ; \Gamma_{j k}\right)=\mu_{i j k l}(L) .
$$

ПРЕДЛОЖЕНИЕ 4.2 (cp. [4]).

1. Предыдущее определение корректно и не зависит от выбора семейства поверхностей Зейферта.

2. Для произвольного четырехкомпонентного полуограничивающего зачепления L справедливы тождества

$$
\begin{gathered}
\mu_{i j k l}(L)+\mu_{i l j k}(L)+\mu_{i k l j}(L)=0, \\
\mu_{i j k l}(L)=-\mu_{j i k l}(L)=\mu_{i j l k}(L)=-\mu_{i j k l}(L), \\
\mu_{i j k l}(L)=\mu_{k l i j}(L) .
\end{gathered}
$$

Как было объяснено в предыдущем пукте, для трехкомпонентных зацеплений есть два типа движений, которые переводят полуограничивающие зацепления в полуограничивающие: $\Delta$-движения и сбалансированные $\times$-движения. Для четырехкомпонентных зацеплений высшим аналогом $\Delta$-движений служат движения, которые приводятся в работе [13]. При этом движении в окрестности точки перестройки расположены все четыре компоненты, так что проекция на плоскость не обладает свойством общего положения, как в случае $\Delta$-движения. Милноровские инварианты третьего порядка изменяются на \pm 1 в зависимости от разновидности движения.

Аналогом сбалансированного $\times$-движения, является сбалансированное $\Delta$-движение, коротко $B \Delta$-движение, котороемы определим следующим образом. $B \Delta$-движение - это пара одновременных $\Delta$-движений, в каждом из которых участвуют ровно три ветви одной компоненты, и знаки у исчезающих треугольников при каждом движении противоположны. Следовательно, каждое сбалансированное $\Delta$-движение переводит полуограничивающее четырехкомпонентное зацепление в полуограничивающее зацепление.

Для формулировки правила об изменении значения инварианта $\mu_{i j k l}$ в ходе сбалансированного $\Delta$-движения вводим следуюшие обозначения: пусть $L_{i}^{+}, L_{j}^{+}$и $L_{k}^{+}$- те ветви кривьх $L_{i}, L_{j}$ и $L_{k}$, у которых начало в положительном исчезающем треугольнике и конец в отрицательном в соответствии с заданной ориентацией компонент $L_{i}, L_{j}$ и $L_{k}$. Пусть $L_{i}^{-}, L_{j}^{-}$и $L_{k}^{-}$- дополнительные ветви.

\section{Teopema 4.3.}

1. Для сбалансированного $\Delta$-двихсения полуограничивающего четырехкомпонентного зацепления $L$, в ходе которого участвуют ветви кривых $L_{i}, L_{j}$, $L_{k}$, значение инварианта $\mu_{i j k l}(L)$ увеличивается на разность соответствующих коэффичиентов зачепления

$$
\operatorname{lk}\left(L_{i}^{+} \cup L_{j}^{-}, L_{l}\right)-\operatorname{lk}\left(L_{i}^{+} \cup L_{k}, L_{l}\right) .
$$

В случае сбалансированного $\Delta$-движсени, в ходе которого участвуют ветви компонент $L_{i^{\prime}}, L_{j^{\prime}}, L_{k^{\prime}}$, значение инварианта изменяется по тому же правилу с учетом знака подстановки $\left(\begin{array}{ccc}i & j & k \\ i^{\prime} & j^{\prime} & k^{\prime}\end{array}\right)$. 
2. При $\Delta$-движении (несбалансированном), в окрестности которого встречаются лишь ветви одной или двух компонент зачепления, значение $\mu_{i j k l}$ не изменяется.

ОпредЕЛЕниЕ 4.4. Скажем, что два четырехкомпонентных зацепления $L$ и $L^{\prime} \Delta-\imath o$ мотопны, если их проекции можно связать последовательностью преобразований Рейдемейстера и $\Delta$-движений. Далее, два четырехкомпонентных зацепления $L$ и $L^{\prime} c \sigma a-$ лансированно $\Delta$-гомотопны, если их проекции можно связать последовательностью преобразований Рейдемейстера, $\Delta$-движений, в которых участвуют не более двух компонент зацепления, и сбалансированных $\Delta$-движений.

ТЕОрема 4.5. Любые два полуограничивающих четырехкомпонентных зацепления являются сбалансировано $\Delta$-гомотопными.

Теорема 4.3 позволяет определить инвариант $\mu_{i j k l}$ аксиоматически, используя формулу (5), определяющую правило пересчета инварианта при изменении зацепления в изотопическом классе. При этом значение инварианта полагается равным нулю для зацепления, компоненты которого заключены в непересекающиеся шары. Единственность инварианта с рассматриваемыми свойствами (2)-(4) вытекает из теоремы 4.5, а его существование вытекает из предложения 4.2 .

5. Окончание доказательств. В этом пункте мы докажем предложения 3.1 и 4.2, теоремы 4.3 и 4.5 .

ДОКАЗАТЕЛЬСТВО ПРЕДЛОЖЕНИЯ 3.1 очевИдно. Подробности можно найти в [8].

ДоКАЗАТЕЛЬСТвО ПРЕДЛОЖЕНИЯ 4.2. Любая поверхность Зейферта $S_{i}, i=1,2$, 3,4 , для соответствующей компоненты зацепления $L$ определяется с однозначно с точностью до вложенного кобордизма и несвязной суммы с семейством вложенных непересекающихся торов, которые охватывают остальные три компоненты зацепления, т.е. каждьй из этих торов является границей малой трубчатой окрестности и наделен той или иной коориентацией. При добавлении одного такого тора, скажем, к примеру, охватывающего $j$-ю компоненту внешним образом, к кривой самопересечения $\Gamma_{i j}$ добавляется компонента, параллельная (с учетом выбора ориентации) компоненте зацепления $L_{j}$. При этом значение коэффициента зацепления $\operatorname{lk}\left(\Gamma_{i j} ; \Gamma_{k l}\right)$ не изменяется, поскольку $\mu_{i j k}(L)=\mu_{i j l}(L)=0$ по условию полуограничиваемости. Следовательно, не изменяется и значение любого $\mu$-инварианта.

Теперь проверим, что при вложенном кобордизме одной из поверхностей Зейферта, скажем $S_{i}$, значение выражения (1) и всех выражений, полученных из него перестановками индексов, не изменяется. Если в процессе кобордизма не встречается точек пересечения всех четырех поверхностей Зейферта, то утверждение очевидно. В точке четырехкратного пересечения изменяются оба коэффициента зацепления, входящие в разность (1), причем на +1 или -1 одновременно. Следовательно, значение $\mu$ не зависит от выбора семейства поверхностей Зейферта. Свойства (2)-(4) вытекают из определения. Предложение доказано.

ДОКАЗАТЕЛЬСТВО ТЕОРЕМЫ 4.3. Если в $\Delta$-дВижении не участвуют три компоненты зацепления, то значения всех коэффициентов зацепления сохраняются. Рассмотрим случай сбалансированного $\Delta$-движения. Для определенности будем считать, что в ходе 
этого преобразования участвуют компоненты $L_{2}, L_{3}, L_{4}$, а изучается поведение инварианта $\mu_{1234}$.

В п. 3 объяснялось, что при одном $\Delta$-движении для трехкомпонентного полуограничивающего зацепления с компонентами $i, j, k$ систему поверхностей Зейферта удобно выбрать так, что после преобразования в окрестности исчезающего треугольника возникает точка тройного пересечения или, иначе, три цикла $\gamma_{i j} \subset \Gamma_{i j}$, каждьй из которых зацеплен с компонентой $L_{k}$ с коэффициентом \pm 1 в зависимости от знака соответствующего исчезающего треугольника при $\Delta$-движении. Аналогично, при сбалансированном $\Delta$-движении возникает пара точек тройного самопересечения, каждая из которых расположена в окрестности каждой точки перестройки пересечения после сбалансированного $\Delta$-движения.

Заметим, что в окрестности пары троек замкнутых циклов на кривых $\Gamma_{23}, \Gamma_{34}, \Gamma_{41}$, каждьй из которых зацеплен с соответствующей компонентой зацепления, расположены, вообще говоря, компоненты кривьх $\Gamma_{13}$ и $\Gamma_{14}$. Выбирая сначала поверхности Зейферта произвольньми (даже допуская пересечения соседних компонент), а затем перестраивая их по трубочкам, как это объяснялось в п. 3, очевидно, можно добиться того, что указанные компоненты располагаются лишь в окрестности одной из особых точек параллельно компоненте $L_{2}$. Алгебраическое число ветвей соответствующих компонент в точности совпадает с коэффициентом зацепления $\operatorname{lk}\left(L_{2}^{+} \cup L_{3}^{-}, L_{1}\right)$ и $\operatorname{lk}\left(L_{2}^{+} \cup L_{4}, L_{1}\right)$, который определяет изменение значения инварианта $\mu_{1234}$ в соответствии с выражением (5). Это доказывает теорему.

ДокАЗАТЕЛЬСТвО ТЕОРЕМЫ 4.5. Мы приводим лишњ краткий набросок доказательства и планируем в следующих публикациях изложить более общую конструкцию. На рис. 3 показано, что $\Delta$-движение может быть реализовано приклейкой борромеевского зацепления внутри исчезающего треугольника и тремя движениями Рейдемейстера второго типа (мы используем терминологию из [15]). Прямой проверкой можно убедиться, что операция приклейки двух борромеевских зацеплений перестановочна между собой с точностью до одновременных приклеек нескольких пар борромеевских зацеплений, имеющих разные знаки, к одинаковьм тройкам компонент и некоторого числа борромеевских зацеплений к одной или двум компонентам зацепления (т.е. одна операция последовательной приклейки двух борромеевских зацеплений переводится в другую при изменении изотопии и некоторого числа описанных вьше движений). Это означает, что, изменяя $\Delta$-гомотопию на сбалансированную $\Delta$-гомотопию, последовательность $\Delta$-движений в исходной гомотопии можно произвольным образом изменить.

Известно, что любые два четырехкомпонентных зацепления с одинаковыми матрицами коэффициентов зацепления пар соответствующих компонент являются $\Delta$-гомотопными (см. [10]), т.е. существует последовательность движений Рейдемейстера и $\Delta$-движений, связьвающая проекции зацеплений. Поскольку зацепления являются полуограничивающими, в силу предложения 3.2 сумма знаков всех $\Delta$-движений, в которых участвуют данные три компоненты, равна нулю. Изменим порядок в последовательности $\Delta$-движений так, что она будет состоять из пар последовательных $\Delta$-движений с разными знаками и $\Delta$-движений, в которых участвуют одна или две компоненты. Требуемая гомотопия является искомой. 


\section{СПИСОК ЦИТИРОВАННОЙ ЛИТЕРАТУРЫ}

[1] Milnor J. W. Link groups // Ann. of Math. 1954. V. 59. № 2. P. 177-195.

[2] Porter R. Milnor $\mu$-invariants and Massey products // Trans. Amer. Math. Soc. 1980. V. 257. P. 39-71.

[3] Тураев В. Г. Инварианты Милнора и произведения Масси // Учеб. записки ЛОМИ. 1976. T. 66 . C. $189-203$.

[4] Cochran T.D. Derivatives of links: Milnor's concordance invariants and Massey's products // Mem. Amer. Math. Soc. 1990. V. 84. № 427. P. 1-73.

[5] Mellor B. Finite Type Homotopy Invariants, I. E-print math .GT/9807162u4, 1999.

[6] Kirk P., Livingston C. Vassiliev invariants of two components links and the Casson-Walker invariant // Topology. 1997. V. 36. P. 1333-1353.

[7] Ахметьев П. М., Реповш Д. Обобщение инварианта Сато-Левина // Тр. МИАН. 1998. T. 221. C. $69-80$.

[8] Cochran T.D. Concordance invariance of the coefficients of Conway's link polynomial // Invent. Math. 1985. V. 82. P. 527-541.

[9] Akhmet'ev P. M., Ruzmaikin A. Borromeanism and Bordism // Topological Aspects of the Dynamics of Fluids and Plasma / ed. H.K. Moffatt et al. Dordrecht: Kluwer, 1992. P. 249-260.

[10] Murakami H., Nakanishi Y. On a certain move generating link-homology // Math. Ann. 1989. V. 284. P. 75-89.

[11] Mellor B. Finite Type Homotopy Invariants, II: Milnor's $\bar{m} u$-Invariants. E-print math .GT/ 9812119, 1998.

[12] Stanford T. Braid Commutators and $\Delta$ Finite-type Invariants. E-print math.GT/9907071, 1999.

[13] Habiro K. Claspers and finite type invariants of links // Geom. Topol. 2000. V. 4. P. 1-83.

[14] Levine J.P. An approach to homotopy classification of links // Trans. Amer. Math. Soc. 1988. V. 306.

[15] Ахметьев П. М., Малешич Й., Реповш Д. Формула для обобщенного инварианта Сато-Левина // Матем. сб. (в печати).

(П. М. Ахметьев) ИЗМИРАН, г. Троицк

Поступило

(Й. Малешич, Д. Реповш) Люблянский университет, Словения

29.11.2000

E-mail: akhmetev@izmiran.rssi.ru, joze.malesic@uni-lj.si,

dusan.repovs@fmf.uni-lj.si 